Forum Math. 10 (1998), 619-639

\title{
Quantum groups and cylinder braiding
}

\section{Tammo tom Dieck and Reinhard Häring-Oldenburg}

(Communicated by Karl H. Hofmann)

\begin{abstract}
The purpose of this paper is to introduce a new structure into the representation theory of quantum groups. The structure is motivated by braid and knot theory. Representations of quantum groups associated to classical Lie algebras have an additional symmetry which cannot be seen in the classical limit. We first explain the general formalism of these symmetries (called cylinder forms) in the context of comodules. Basic ingredients are tensor representations of braid groups of type $B$ derived from standard $R$-matrices associated to socalled four braid pairs. These are applied to the Faddeev-Reshetikhin-Takhtadjian construction of bialgebras from $R$-matrices. As a consequence one obtains four braid pairs on all representations of the quantum group. In the second part of the paper we study in detail the dual situation of modules over the quantum enveloping algebra $U_{q}\left(s l_{2}\right)$. The main result here is the computation of the universal cylinder twist.
\end{abstract}

1991 Mathematics Subject Classification: 17B37, 18D10, 57M25.

\section{Cylinder forms}

Let $A=(A, m, e, \mu, \varepsilon)$ be a bialgebra over the commutative ring $\Omega$ with multiplication $m$, unit $e$, comultiplication $\mu$, and counit $\varepsilon$. Let $r: A \otimes A \rightarrow \Omega$ be a linear form. We associate to left $A$-comodules $M, N$ the $\mathfrak{R}$-linear map

$$
z_{M, N}: M \otimes N \rightarrow N \otimes M, \quad x \otimes y \mapsto \sum r\left(y^{1} \otimes x^{1}\right) y^{2} \otimes x^{2}
$$

where we have used the symbolic notation $x \mapsto \sum x^{1} \otimes x^{2}$ for a left $A$-comodule structure $\mu_{M}: M \rightarrow A \otimes M$ on $M$. We call $r$ a braid form on $A$, if the $z_{M, N}$ yield a braiding on the tensor category $A$-Com of left $A$-comodules. We refer to [7, Def. VIII.5.1 on p.184] for the properties of $r$ which make it into a braid form.

Let $(C, \mu, \varepsilon)$ be a coalgebra. We use symbolic notations like $\mu(a)=\sum a_{1} \otimes a_{2}$ and $(\mu \otimes 1) \mu(a)=\mu_{2}(a)=\sum a_{1} \otimes a_{2} \otimes a_{3}=\sum a_{11} \otimes a_{12} \otimes a_{2}$ for the comultiplication. The multiplication in the dual algebra $C^{*}$ is denoted as convolution: If 
$f, g \in C^{*}$ are $\mathcal{R}$-linear forms on $C$, then the convolution $f * g$ is the form defined by $a \mapsto \sum f\left(a_{1}\right) g\left(a_{2}\right)$. The unit element of the algebra $C^{*}$ is $\varepsilon$. Therefore $g$ is a (convolution) inverse of $f$, if $f * g=g * f=\varepsilon$. We apply this formalism to the coalgebras $A$ and $A \otimes A$. If $f$ and $g$ are linear forms on $A$, we denote by $f \hat{\otimes} g$ the linear form on $A \otimes A$ defined by $a \otimes b \mapsto f(a) g(b)$. The twist on $A \otimes A$ is $\tau(a \otimes b)=b \otimes a$.

Here is the main definition of this paper. Let $(A, r)$ be a bialgebra with braid form $r$. A linear form $f: A \rightarrow \Omega$ is called a cylinder form for $(A, r)$, if it is convolution invertible and satisfies

$$
f \circ m=(f \hat{\otimes} \varepsilon) * r \tau *(\varepsilon \hat{\otimes} f) * r=r \tau *(\varepsilon \hat{\otimes} f) * r *(f \hat{\otimes} \varepsilon) .
$$

In terms of elements and symbolic notation, (1.1) assumes the following form:

(1.2) For any two elements $a, b \in A$ the identities

$$
\begin{aligned}
f(a b) & =\sum f\left(a_{1}\right) r\left(b_{1} \otimes a_{2}\right) f\left(b_{2}\right) r\left(a_{3} \otimes b_{3}\right) \\
& =\sum r\left(b_{1} \otimes a_{1}\right) f\left(b_{2}\right) r\left(a_{2} \otimes b_{3}\right) f\left(a_{3}\right)
\end{aligned}
$$

hold.

A cylinder form (in fact any linear form) yields for each left $A$-comodule $M$ a R-linear endomorphism

$$
t_{M}: M \rightarrow M, \quad x \mapsto \sum f\left(x^{1}\right) x^{2} .
$$

If $\varphi: M \rightarrow N$ is a morphism of comodules, then $\varphi \circ t_{M}=t_{N} \circ \varphi$. Since $t_{M}$ is in general not a morphism of comodules we express this fact by saying: The $t_{M}$ constitute a weak endomorphism of the identity functor of $A$-Com. We call $t_{M}$ the cylinder twist on $M$. The axiom (1.1) for a cylinder form has the following consequence.

(1.3) Proposition. The linear map $t_{M}$ is invertible. For any two comodules $M, N$ the identities

$$
t_{M \otimes N}=z_{N, M}\left(t_{N} \otimes 1_{M}\right) z_{M, N}\left(t_{M} \otimes 1_{N}\right)=\left(t_{M} \otimes 1_{N}\right) z_{N, M}\left(t_{N} \otimes 1_{M}\right) z_{M, N}
$$

hold.

Proof. Let $g$ be a convolution inverse of $f$. Set $s_{M}: M \rightarrow M, x \mapsto \sum g\left(x^{1}\right) x^{2}$. Then

$$
s_{M} t_{M}(x)=\sum f\left(x^{1}\right) g\left(x^{21}\right) x^{22}=\sum \varepsilon\left(x^{1}\right) x^{2}=x,
$$

by the definition of the convolution inverse and the counit axiom. Hence $s_{M}$ is inverse to $t_{M}$. 
In order to verify the second equality, we insert the definitions and see that the second map is

$$
x \otimes y \mapsto \sum f\left(x^{1}\right) r\left(y^{1} \otimes x^{21}\right) f\left(y^{21}\right) r\left(y^{221} \otimes x^{221}\right) y^{222} \otimes x^{222}
$$

while the third map is

$$
x \otimes y \mapsto \sum r\left(y^{1} \otimes x^{1}\right) f\left(y^{21}\right) r\left(y^{21} \otimes x^{221}\right) f\left(x^{221}\right) y^{222} \otimes x^{222} .
$$

The coassociativity of the comodule structure yields a rewriting of the form

$$
\sum y^{1} \otimes y^{21} \otimes y^{221} \otimes y^{222}=\sum\left(y^{1}\right)_{1} \otimes\left(y^{1}\right)_{2} \otimes\left(y^{1}\right)_{3} \otimes y^{2}
$$

and similarly with $y$ replaced by $x$. We now apply (1.2) in the case where $(a, b)=\left(x^{1}, y^{1}\right)$.

By definition of the comodule structure of $M \otimes N$, the map $t_{M \otimes N}$ has the form $x \otimes y \mapsto \sum f\left(x^{1} y^{1}\right) x^{2} \otimes y^{2}$. Again we use (1.2) in the case where $(a, b)=\left(x^{1}, y^{1}\right)$ and obtain the first equality of (1.3).

We also mention dual notions. Let $A$ be a bialgebra with a universal $R$-matrix $R \in A \otimes A$. An element $v \in A$ is called a (universal) cylinder twist for $(A, R)$, if it is invertible and satisfies

$$
\mu(v)=(v \otimes 1) \cdot \tau R \cdot(1 \otimes v) \cdot R=\tau R \cdot(1 \otimes v) \cdot R \cdot(v \otimes 1)
$$

The $R$-matrix $R=\sum a_{r} \otimes b_{r}$ induces the braiding $z_{M, N}: M \otimes N \rightarrow N \otimes M$, $x \otimes y \mapsto \sum b_{r} y \otimes a_{r} x$. Let $t_{M}: M \rightarrow M, x \mapsto v x$ be the induced cylinder twist. Again the $t_{M}$ form a weak endomorphism of the identity functor. If $v$ is not central in $A$, then the $t_{M}$ are not in general $A$-module morphisms. The relations (1.3) also holds in this context.

If a ribbon algebra is defined as in [7, p. 361], then the element $\theta^{-1}$, loc. cit., is a cylinder twist in the sense above.

\section{Tensor representations of braid groups}

The braid group $Z B_{n}$ associated to the Coxeter graph $B_{n}$

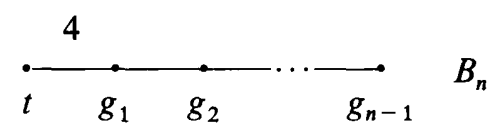

with $n$ vertices has generators $t, g_{1}, \ldots, g_{n-1}$ and relations:

$$
\begin{aligned}
t g_{1} t g_{1} & =g_{1} t g_{1} t \\
t g_{i} & =g_{i} t \quad i>1
\end{aligned}
$$




$$
\begin{array}{ccrl}
g_{i} g_{j} & =g_{j} g_{i} & & |i-j| \geq 2 \\
g_{i} g_{j} g_{i} & =g_{j} g_{i} g_{j} & & |i-j|=1
\end{array}
$$

We recall: The group $Z B_{n}$ is the group of braids with $n$ strings in the cylinder $(\mathbb{C} \backslash 0) \times[0,1]$ from $\{1, \ldots, n\} \times 0$ to $\{1, \ldots, n\} \times 1$. This topological interpretation is the reason for using the cylinder terminology. For the relation between the root system $B_{n}$ and $Z B_{n}$ see [2].

Let $V$ be a $\Re$-module. Suppose $X: V \otimes V \rightarrow V \otimes V$ and $F: V \rightarrow V$ are $\Re$-linear automorphisms with the following properties:

(1) $X$ is a Yang-Baxter operator, i.e., $X$ satisfies the equation

$$
(X \otimes 1)(1 \otimes X)(X \otimes 1)=(1 \otimes X)(X \otimes 1)(1 \otimes X)
$$

on $V \otimes V \otimes V$.

(2) With $Y=F \otimes 1_{V}$, the four braid relation $Y X Y X=X Y X Y$ is satisfied.

If (1) and (2) hold, we call $(X, F)$ a four braid pair. For the construction of four braid pairs associated to standard $R$-matrices see [4]. For a geometric interpretation of (2) in terms of symmetric braids with 4 strings see [3].

Given a four braid pair $(X, F)$, we obtain a tensor representation of $Z B_{n}$ on the $n$-fold tensor power $V^{\otimes n}$ of $V$ by the following assignment:

$$
\begin{aligned}
t & \mapsto F \otimes 1 \otimes \cdots \otimes 1 \\
g_{i} & \mapsto X_{i}=1 \otimes \cdots \otimes X \otimes \cdots \otimes 1
\end{aligned}
$$

The $X$ in $X_{i}$ acts on the factors $i$ and $i+1$.

These representations give rise to further operators, if we apply them to special elements in the braid groups. We set

$$
\begin{aligned}
& t(1)=t, \quad t(j)=g_{j-1} g_{j-2} \cdots g_{1} t g_{1} g_{2} \cdots g_{j-1}, \quad t_{n}=t(1) t(2) \cdots t(n) \\
& g(j)=g_{j} g_{j+1} \cdots g_{j+n-1}, \quad x_{m, n}=g(m) g(m-1) \cdots g(1) .
\end{aligned}
$$

The elements $t(j)$ pairwise commute. We denote by $T_{n}: V^{\otimes n} \rightarrow V^{\otimes n}$ and by $X_{m, n}: V^{\otimes m} \otimes V^{\otimes n} \rightarrow V^{\otimes n} \otimes V^{\otimes m}$, respectively, the operators induced by $t_{n}$ and by $x_{m, n}$, respectively.

(2.3) Proposition. The following identities hold

$$
T_{m+n}=X_{n, m}\left(T_{n} \otimes 1\right) X_{m, n}\left(T_{m} \otimes 1\right)=\left(T_{m} \otimes 1\right) X_{n, m}\left(T_{n} \otimes 1\right) X_{m, n}
$$

Proof. We use some facts about Coxeter groups [1, CH. IV, §1]. If we adjoin the relations $t^{2}=1$ and $g_{j}^{2}=1$ to (2.1) we obtain the Coxeter group $C B_{n}$. The element $t_{n}$ is given as a product of $n^{2}$ generators $t, g_{j}$. The unique longest element of $C B_{n}$ has length $n^{2}$ and is equal to $t_{n}$. The element $x_{n, m} t_{n} x_{m, n} t_{m}$ of $C B_{m+n}$ has length 
$(m+n)^{2}$ and therefore equals $t_{m+n}$ in $C B_{m+n}$. By a fundamental fact about braid groups [1, CH. IV, §1.5, Prop. 5], the corresponding elements in the braid group are equal. We now apply the tensor representation and obtain the first equality of (2.3).

For later use we record:

(2.4) Proposition. The element $t_{n}$ is contained in the center of $Z B_{n}$.

\section{Cylinder forms from four braid pairs}

Let $V$ be a free $\Re$-module with basis $v_{1}, \ldots, v_{n}$. Associated to a Yang-Baxter operator $X: V \otimes V \rightarrow V \otimes V$ is a bialgebra $A=A(V, X)$ with braid form $r$ obtained via the FRT-construction (see [7, VIII.6] for the construction of $A$ and $r$ ). We show that a four braid pair $(X, F)$ induces a canonical cylinder form on $(A, r)$.

Recall that $A$ is a quotient of a free algebra $\tilde{A}$. We use the model

$$
\bigoplus_{n=0}^{\infty} \operatorname{Hom}\left(V^{\otimes n}, V^{\otimes n}\right)=\tilde{A}
$$

The multiplication in $\tilde{A}$ is given by the canonical identification $E_{k} \otimes E_{l} \cong E_{k+l}$, $f \otimes g \mapsto f \otimes g$, with $E_{k}=\operatorname{Hom}\left(V^{\otimes k}, V^{\otimes k}\right)$. The canonical basis $T_{i}^{j}: v_{k} \mapsto \delta_{i, k} v_{j}$ of $E_{1}$ induces the basis

$$
T_{i}^{j}=T_{i_{1}}^{j_{1}} \otimes \cdots \otimes T_{i_{k}}^{j_{k}}
$$

of $E_{k}$, with multi-index notation $i=\left(i_{1}, \ldots, i_{k}\right), j=\left(j_{1}, \ldots, j_{k}\right)$. The comultiplication in $\tilde{A}$ is given by $\mu\left(T_{i}^{j}\right)=\sum_{k} T_{i}^{k} \otimes T_{k}^{j}$ and the counit by $\varepsilon\left(T_{i}^{j}\right)=\delta_{i}^{j}$.

In section 2 we defined an operator $T_{k} \in E_{k}$ from a given four braid pair $(X, F)$. We express $T_{k}$ in terms of our basis

$$
T_{k}\left(v_{i}\right)=\sum_{j} F_{i}^{j} v_{j}
$$

again using the multi-index notation $v_{i}=v_{i_{1}} \otimes \cdots \otimes v_{i_{k}}$ when $i=\left(i_{1}, \ldots, i_{k}\right)$. We define a linear form

$$
\tilde{f}: \tilde{A} \rightarrow \Re, \quad T_{i}^{j} \mapsto F_{i}^{j} .
$$

(3.1) Theorem. The linear form $\tilde{f}$ factors through the quotient map $\tilde{A} \rightarrow A$ and induces a cylinder form $f$ for $(A, r)$.

Proof. Suppose the operator $X=X_{m, n}: V^{\otimes m} \otimes V^{\otimes n} \rightarrow V^{\otimes n} \otimes V^{\otimes m}$ has the form $X\left(v_{i} \otimes v_{j}\right)=\sum_{a b} X_{i j}^{a b} v_{a} \otimes v_{b}$. We define a form $\tilde{r}: \tilde{A} \otimes \tilde{A} \rightarrow \Re$ by 


$$
\tilde{r}: E_{k} \otimes E_{l} \rightarrow \Re, \quad T_{i}^{a} \otimes T_{j}^{b} \mapsto X_{j i}^{a b}
$$

The form $\tilde{r}$ factors through the quotient $A \otimes A$ and induces $r$.

Claim: The forms $\tilde{r}$ and $\tilde{f}$ satisfy (1.1) and (1.2). Proof of the claim: In the proof we use the summation convention: summation over an upper-lower index. Then we can write $\mu_{2}\left(T_{i}^{c}\right)=T_{i}^{k} \otimes T_{k}^{a} \otimes T_{a}^{c}$ and $\mu_{2}\left(T_{j}^{d}\right)=T_{j}^{l} \otimes T_{l}^{b} \otimes T_{b}^{d}$. The equality (1.2) amounts to

$$
F_{i j}^{c d}=F_{i}^{k} X_{k j}^{l a} F_{l}^{b} X_{b a}^{c d}=X_{i j}^{l k} F_{l}^{b} X_{b k}^{a d} F_{a}^{c}
$$

These equations are also a translation of (2.3) into matrix form. This finishes the proof of the claim.

We have to show that $\tilde{f}$ maps the kernel $I$ of the projection $\tilde{A} \rightarrow A$ to zero. But this is a consequence of (1.2), applied in the case $b=1$, since one of the terms $a_{1}, a_{2}, a_{3}$ is contained in $I$ and $\tilde{r}$ is the zero map on $I \otimes \tilde{A}$ and $\tilde{A} \otimes I$.

It remains to show that $f$ is convolution invertible. The pair $\left(X^{-1}, F^{-1}\right)$ is a four braid pair. Let $\bar{r}$ and $\bar{f}$ be the induced operators on $\tilde{A}$. Then $\tilde{f} * \bar{f}=\varepsilon=\bar{f} * \bar{f}$ on $\tilde{A}$, and (1.2) holds for $(\bar{f}, \vec{r})$ in place of $(f, r)$. The Yang-Baxter operator $X^{-1}$ defines the same quotient $A$ of $\tilde{A}$ as $X$. Hence the kernel ideal obtained from $X^{-1}$ equals $I$, and therefore $\bar{f}(I)=0$.

We have the comodule $V \rightarrow A \otimes V, v_{i} \mapsto \sum_{j} T_{i}^{j} \otimes v_{j}$, and similarly for $V^{\otimes k}$ using multi-index notation. By construction we have:

(3.2) Proposition. The cylinder form f induces on $V^{\otimes k}$ the cylinder twist $t_{V^{\otimes k}}=T_{k}$.

\section{Tensor categories with cylinder braiding}

The results of the previous section have the following categorical structure:

(1) $\mathscr{B}$ is a category;

(2) $\mathscr{A}$ is a subcategory with the same objects;

(3) The category $\mathscr{A}$ carries the structure of a tensor category with a braiding $z_{M, N}$;

(4) $\mathscr{B}$ is a right tensor module category over $\mathscr{A}$;

(5) For each object $V$ an automorphism $t_{V}: V \rightarrow V$ in $\mathscr{B}$ is given. The $t_{V}$ constitute a weak endomorphism of the identity functor of $\mathscr{A}$.

(6) For each pair $M, N$ of objects the identities (1.3) hold.

The meaning of (4) is the following: There is given a functor $\otimes: \mathscr{B} \times \mathscr{A} \rightarrow \mathscr{B}$ and a natural associativity isomorphism $a: A \otimes(B \otimes C) \rightarrow(A \otimes B) \otimes C$ of functors $\mathscr{B} \times \mathscr{A} \times \mathscr{A} \rightarrow \mathscr{B}$. The pentagon axiom of tensor category theory (which still makes sense in this context) is also assumed. The tensor product functor and the associativity $a$ restrict to the given tensor product and associativity in the tensor category $\mathscr{A}$. The unit object of $\mathscr{A}$ is a left and right unit for $\otimes: \mathscr{B} \times \mathscr{A} \rightarrow \mathscr{B}$ and the triangle 
axiom holds. An example of this type of module category arises from a tensor category $\mathscr{B}$ and a tensor subcategory $\mathscr{A}$. (See $[7$, XI.2] for such notions.)

We considered the case where $\mathscr{B}$ was the category of $A$-comodules and $\mathfrak{R}$-linear maps and $\mathscr{A}$ the category of $A$-comodules and $A$-linear maps. (5) is induced by a cylinder form.

The prototype is given by the braid categories themselves. The objects are the natural numbers $n \in \mathbb{N}_{0}$. The morphisms in $\mathscr{B}$ from $n$ to $n$ are the elements in $Z B_{n}$ with composition the group multiplication. There are no morphisms from $m$ to $n$ for $m \neq n$. The morphisms in $\mathscr{A}$ from $n$ to $n$ are the elements of the Artin braid group $Z A_{n-1}$, the subgroup of $Z B_{n}$ generated by $g_{1}, \ldots, g_{n-1}$. The tensor product is given on objects as $m \otimes n=m+n$ and on morphisms as the following homomorphism $Z B_{m} \times Z B_{n} \rightarrow Z B_{m+n}$

$$
\begin{aligned}
& t, g_{1}, \ldots, g_{m-1} \in Z B_{m} \mapsto t, g_{1}, \ldots, g_{m-1} \in Z B_{m+n} \\
& t, g_{1}, \ldots, g_{n-1} \in Z B_{n} \\
& \qquad g_{m} g_{m-1} \cdots g_{1} t g_{1} g_{2} \cdots g_{m}, g_{m+1}, \ldots, g_{m+n-1} \in Z B_{m+n} .
\end{aligned}
$$

The braiding is given by the morphisms $x_{m, n}$ of section 2 and the morphisms $t_{n}$ are also specified in that section. By (2.4), the $t_{n}$ constitute an endomorphism of the identity of $\mathscr{B}$.

There is a natural quotient category of this braid category (when $R$-linearized), namely the Temperley-Lieb category of type $B$ via the Kauffman functor (see [3]).

For an elaboration of the categorical viewpoint and applications to knot theory along the lines of [9] see [5] and [6].

\section{The example $\mathrm{SL}_{q}(2)$}

We illustrate the theory with the quantum group associated to $S L_{2}$. For simplicity we work over the function field $\mathbb{Q}\left(q^{1 / 2}\right)=\mathfrak{R}$.

Let $V$ be a two-dimensional $\mathcal{R}$-module with basis $v_{1}, v_{2}$. In terms of the basis $v_{1} \otimes v_{1}, v_{1} \otimes v_{2}, v_{2} \otimes v_{1}, v_{2} \otimes v_{2}$ the matrix

$$
X=q^{-1 / 2}\left(\begin{array}{ccccc}
q & & & \\
& q-q^{-1} & 1 & \\
& 1 & 0 & \\
& & & q
\end{array}\right)
$$

defines a Yang-Baxter operator. The FRT-construction associates to $X$ the algebra $A$ generated by $a, b, c, d=T_{1}^{1}, T_{1}^{2}, T_{2}^{1}, T_{2}^{2}$ with relations 


$$
\begin{array}{rl}
a b=q b a & b d=q d b \\
a c=q c a & c d=q d c \\
b c= & c b \\
a d-d a= & \left(q-q^{-1}\right) b c .
\end{array}
$$

The matrix

$$
F=\left(\begin{array}{ll}
0 & \beta \\
\alpha & \theta
\end{array}\right)
$$

yields a four braid pair $(X, F)$ for arbitrary parameters with invertible $\alpha \beta$ (see [4], also for an $n$-dimensional generalization). The quantum plane $P=\Re\{x, y\}$ / $(x y-q y x)$ is a left $A$-comodule via the map $\mu_{P}: P \rightarrow A \otimes P$ given by

$$
\mu_{P}\left(x^{i} y^{j}\right)=\sum_{r=0}^{i} \sum_{s=0}^{j} q^{-s(i+j-r-s)-r(i-r)}\left[\begin{array}{l}
i \\
r
\end{array}\right]\left[\begin{array}{l}
j \\
s
\end{array}\right] a^{r} b^{i-r} c^{s} d^{j-s} \otimes x^{r+s} y^{i+j-r-s}
$$

where $\left[\begin{array}{l}i \\ r\end{array}\right]$ is a $q$-binomial coefficient

$$
\left[\begin{array}{l}
i \\
r
\end{array}\right]=\frac{[i] !}{[r] ![i-r] !}, \quad[i] !=[1][2] \cdots[i], \quad[i]=\frac{q^{i}-q^{-i}}{q-q^{-1}}
$$

(compare [7, IV], where different conventions are used).

The operator $T_{2}=(F \otimes 1) X(F \otimes 1) X$ on $V \otimes V$ has the matrix (with $\delta=q-q^{-1}$ )

$$
\left(\begin{array}{cccc}
0 & 0 & 0 & \beta^{2} \\
0 & \alpha \beta \delta & \alpha \beta & q \beta \theta \\
0 & \alpha \beta & 0 & \beta \theta \\
\alpha^{2} & q \alpha \theta & \alpha \theta & \alpha \beta \delta+q \theta^{2}
\end{array}\right)=\left(\begin{array}{cccc}
F_{11}^{11} & F_{12}^{11} & F_{21}^{11} & F_{22}^{11} \\
F_{11}^{12} & F_{12}^{12} & F_{21}^{12} & F_{22}^{12} \\
F_{11}^{21} & F_{12}^{21} & F_{21}^{21} & F_{22}^{21} \\
F_{11}^{22} & F_{12}^{22} & F_{21}^{22} & F_{22}^{22}
\end{array}\right)
$$

with respect to the basis $v_{1} \otimes v_{1}, v_{1} \otimes v_{2}, v_{2} \otimes v_{1}, v_{2} \otimes v_{2}$. This is also the matrix of values of the cylinder form $f$

$$
f\left(\begin{array}{llll}
a a & a c & c a & c c \\
a b & a d & c b & c d \\
b a & b c & d a & d c \\
b b & b d & d b & d d
\end{array}\right)
$$

Let $\operatorname{det}_{q}=a d-q b c$ be the quantum determinant. It is a group-like central element of $A$. The quotient of $A$ by the ideal generated by $\operatorname{det}_{q}$ is the Hopf algebra $S L_{q}(2)$. 
(5.3) Proposition. The form $f$ has the value $-q^{-1} \alpha \beta$ on $\operatorname{det}_{q}$. If $-q^{-1} \alpha \beta=1$, then f factors over $S L_{q}(2)$.

Proof. The stated value of $f\left(\operatorname{det}_{q}\right)$ is computed from the data above. We use the fact that

$$
r\left(x \otimes \operatorname{det}_{q}\right)=r\left(\operatorname{det}_{q} \otimes x\right)=\varepsilon(x),
$$

see [7, p. 195]. From (1.2) we obtain, for $a \in A$ and $b=\operatorname{det}_{q}$, that

$$
\begin{aligned}
f(a b) & =\sum f\left(a_{1}\right) r\left(b_{1} \otimes a_{2}\right) f\left(b_{2}\right) r\left(a_{3} \otimes b_{3}\right) \\
& =\sum f\left(a_{1}\right) \varepsilon\left(a_{2}\right) f\left(\operatorname{det}_{q}\right) \varepsilon\left(a_{3}\right) \\
& =f(a),
\end{aligned}
$$

by using the assumption that $f\left(\operatorname{det}_{q}\right)=1$ together with the counit axiom.

We consider the subspace $W=V_{2}$ of the quantum plane generated by $x^{2}, x y, y^{2}$. We have

$$
\begin{aligned}
& \mu_{P}\left(x^{2}\right)=b^{2} \otimes y^{2}+\left(1+q^{-2}\right) a b \otimes x y+a^{2} \otimes x^{2} \\
& \mu_{P}(x y)=b d \otimes y^{2}+\left(a d+q^{-1} b c\right) \otimes x y+a c \otimes x^{2} \\
& \mu_{P}\left(y^{2}\right)=d^{2} \otimes y^{2}+\left(1+q^{-2}\right) c d \otimes x y+c^{2} \otimes x^{2} .
\end{aligned}
$$

This yields the following matrix for $t_{W}$ with respect to the basis $x^{2}, x y, y^{2}$

$$
\left(\begin{array}{ccc}
0 & 0 & \beta^{2} \\
0 & q \alpha \beta & \left(q+q^{-1}\right) \beta \theta \\
\alpha^{2} & q \alpha \theta & \alpha \beta \delta+q \theta^{2}
\end{array}\right) .
$$

In the Clebsch-Gordan decomposition $V \otimes V=V_{2} \otimes V_{0}$ the subspace $V_{0}$ (the trivial irreducible module) is spanned by $u=v_{2} \otimes v_{1}-q^{-1} v_{1} \otimes v_{2}$. This is the eigenvector of $X$ with eigenvalue $-q^{-3 / 2}$. It is mapped by $T_{2}$ to $-q^{-1} \alpha \beta u$. If we require this to be the identity we must have $\alpha \beta=-q$. We already obtained this condition by considering the quantum determinant.

The matrix of $t_{W}$ with respect to the basis $w_{1}=x^{2}, w_{2}=\sqrt{1+q^{-2}} x y, w_{3}=y^{2}$ is

$$
F_{2}=\left(\begin{array}{ccc}
0 & 0 & \beta^{2} \\
0 & q \alpha \beta & \sqrt{1+q^{2}} \beta \theta \\
\alpha^{2} & \sqrt{1+q^{2}} \alpha \theta & \alpha \beta \delta+q \theta^{2}
\end{array}\right) .
$$

In case $\alpha=\beta$ this matrix is symmetric. 
The $R$-matrix $X$ on $W \otimes W$ with respect to the lexicographic basis $w_{i} \otimes w_{j}$ with $w_{1}=x^{2}, w_{2}=\sqrt{1+q^{-2}} x y, w_{3}=y^{2}$ has the form

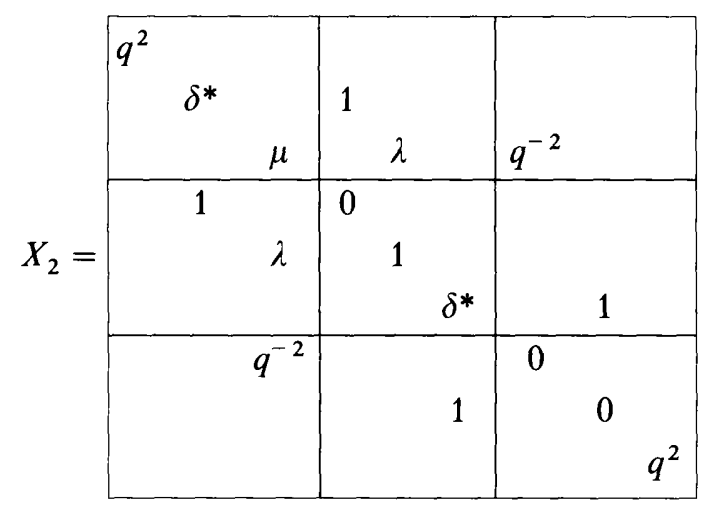

It uses $\delta^{*}=q^{2}-q^{-2}, \mu=\delta^{*}\left(1-q^{-2}\right), \lambda=q^{-1} \delta^{*}$. By construction, $\left(X_{2}, F_{2}\right)$ is a four braid pair.

One now has the problem of computing $t_{W}$ on irreducible comodules $W$. We treat instead the more familiar dual situation of modules over the quantized universal enveloping algebra.

\section{The cylinder braiding for $U$-modules}

The construction of the cylinder form is the simplest method to produce a universal operator for the cylinder twist. In order to compute the cylinder twist explicitly we pass to the dual situation of the quantized universal enveloping algebra $U$. One can formally dualize comodules to modules and thus obtain a cylinder braiding for suitable classes of $U$-modules from the results of the previous sections. But we rather start from scratch.

We work with the Hopf algebra $U=U_{q}\left(s l_{2}\right)$ as in [8]. It is the associative algebra over the function field $\mathbb{Q}\left(q^{1 / 2}\right)=\Omega$ generated ${ }^{1}$ by $K, K^{-1}, E, F$ with relations $K K^{-1}=K^{-1} K=1, K E=q^{2} E K, K F=q^{-2} F K, E F-F E=\left(K-K^{-1}\right) /\left(q-q^{-1}\right)$, comultiplication $\mu(K)=K \otimes K, \mu(E)=E \otimes 1+K \otimes E, \mu(F)=F \otimes K^{-1}+1 \otimes F$, and counit $\varepsilon(K)=1, \varepsilon(E)=\varepsilon(F)=0$. A left $U$-module $M$ is called integrable if the following holds:

(1) $M=\bigoplus M^{n}$ is the direct sum of weight spaces $M^{n}$ on which $K$ acts as multiplication by $q^{n}$ for $n \in \mathbb{Z}$.

(2) $E$ and $F$ are locally nilpotent on $M$.

Let $U$-Int denote the category of integrable $U$-modules and $U$-linear maps. (It would be sufficient to consider only finite dimensional such modules.) An integrable $U$-module $M$ is semi-simple: It has a unique isotypic decomposition $M=\bigoplus_{n \geq 0} M(n)$,

1 There is another use of the letter $F$. It has nothing to do with the $2 \times 2$-matrix $F$ in (5.2). 
with $M(n)$ isomorphic to a direct sum of copies of the irreducible module $V_{n}$. The module $V_{n}$ has a $\Re$-basis $x_{0}, x_{1}, \ldots, x_{n}$ with $F\left(x_{i}\right)=[i+1] x_{i+1}, E\left(x_{i}\right)=$ $[n-i+1] x_{i-1}, x_{-1}=0, x_{n+1}=0$; moreover, $x_{i} \in V_{n}^{n-2 i}$. The category of integrable $U$-modules is braided. The braiding is induced by the universal $R$-matrix $R=\kappa \circ \Psi$ with

$$
\Psi=\sum_{n \geq 0} q^{n(n-1) / 2} \frac{\left(q-q^{-1}\right)^{n}}{[n] !} F^{n} \otimes E^{n}
$$

and $\kappa=q^{H \otimes H / 2}$. Note that $\Psi$ is a well-defined operator on integrable $U$-modules. (This operator is called $\bar{\Theta}$ in $\left[8\right.$, section 4.1] and $L_{i}^{\prime}$ in $[8$, p. 46].) The operator $\kappa$ acts on $M^{m} \otimes N^{n}$ as multiplication by $q^{m n / 2}$. If we view $H$ as the operator $H: M^{m} \rightarrow M^{m}, x \mapsto m x$, then $q^{H \otimes H / 2}$ is a suggestive notation for $\kappa$. The braiding $z_{M, N}: M \otimes N \rightarrow N \otimes M$ is $\tau \circ R$, i.e., the action of $R$ followed by the interchange operator $\tau: x \otimes y \mapsto y \otimes x$.

A four braid pair $(X, F)$ on the vector space $V$ yields a tensor representation of $Z B_{n}$ on $V^{\otimes n}$. We start with the standard four braid pair (5.1) and (5.2) on the twodimensional $U$-module $V=V_{1}$. Let $T_{n}: V^{\otimes n} \rightarrow V^{\otimes n}$ be the associated cylinder twist as defined in section 2. By the Clebsch-Gordan decomposition, $V_{n}$ is contained with multiplicity 1 in $V^{\otimes n}$. Similarly, $V_{m+n} \subset V_{m} \otimes V_{n}$ with multiplicity one [7, VII.7].

(6.2) Lemma. There exists a projection operator $e_{n}: V^{\otimes n} \rightarrow V^{\otimes n}$ whose image, $V_{n}$, commutes with $T_{n}$.

Proof. Let $H_{n}$ be the Hecke algebra over $\Omega$ generated by $x_{1}, \ldots, x_{n-1}$ with braid relations $x_{i} x_{j} x_{i}=x_{j} x_{i} x_{j}$ for $|i-j|=1$ and $x_{j} x_{i}=x_{i} x_{j}$ for $|i-j|>1$ and quadratic relations $\left(x_{i}+1\right)\left(x_{i}-q^{2}\right)=0$. Since $X$ satisfies $\left(X-q^{1 / 2}\right)\left(X+q^{-3 / 2}\right)=0$, we obtain from the action of $Z A_{n-1} \subset Z B_{n}$ on $V^{\otimes n}$ an action of $H_{n}$ if we let $x_{i}$ act as $q^{3 / 2} g_{i}$. Since $T_{n}$ comes from a central element of $Z B_{n}$, see (2.4), the $H_{n}$-action commutes with $T_{n}$. It is well known that there exists an idempotent $e_{n} \in H_{n}$ for which $e_{n} V^{\otimes n}=V_{n}$ (quantized Schur-Weyl duality). This fact implies the assertion of the Lemma.

(6.3) Corollary. The subspace $V_{n} \subset V^{\otimes n}$ is $T_{n}$-stable.

A similar proof shows that all summands in the isotypic decomposition of $V^{\otimes_{n}}$ are $T_{n}$-stable.

We denote by $\tau_{n}$ the restriction of $T_{n}$ to $V_{n}$. On $V_{m} \otimes V_{n}$ we have the induced operator $\tau_{m, n}=z_{n, m}\left(\tau_{n} \otimes 1\right) z_{m, n}\left(\tau_{m} \otimes 1\right)$ where $z_{m, n}$ denotes the braiding on $V_{m} \otimes V_{n}$.

(6.4) Lemma. The subspace $V_{m+n} \subset V_{m} \otimes V_{n}$ is $\tau_{m, n}$-stable. The induced morphism equals $\tau_{m+n}$. 
Proof. Consider $V_{m} \otimes V_{n} \subset V^{\otimes m} \otimes V^{\otimes n}=V^{\otimes(m+n)}$. The projection operator $e_{m} \otimes e_{n}$ is again obtained from the action of a certain element of the Hecke algebra $H_{m+n}$. Hence $V_{m} \otimes V_{n}$ is $T_{m+n}$-stable and the action on the subspace $V_{m+n}$ is $\tau_{m+n}$. We now use the equality (2.3)

$$
T_{m+n}=X_{n, m}\left(T_{n} \otimes 1\right) X_{m, n}\left(T_{m} \otimes 1\right)
$$

The essential fact is that $X_{m, n}$ is the braiding on $V^{\otimes m} \otimes V^{\otimes n}$. It induces, by naturality of the braiding, the braiding $z_{m, n}$ on $V_{m} \otimes V_{n}$.

Let $A(n)=\left(\alpha_{i}^{j}(n)\right)$ be the matrix of $\tau_{n}$ with respect to $x_{0}, \ldots, x_{n}$. In the next theorem we derive a recursive description of $A(n)$. We need more notation to state it. Define inductively polynomials $\gamma_{k}$ by $\gamma_{-1}=0, \gamma_{0}=1$ and, for $k>0$,

$$
\alpha \gamma_{k+1}=q^{k} \theta \gamma_{k}+\beta q^{k-1} \delta[k] \gamma_{k-1}
$$

Here $\gamma_{k}=\gamma_{k}(\theta, q, \alpha, \beta)$ is a polynomial in $\theta$ with coefficients in $\mathbb{Z}\left[q, q^{-1}, \alpha^{-1}, \beta\right]$ and $\delta=q-q^{-1}$. Let $D(n)$ denote the codiagonal matrix with $\alpha^{k} \beta^{n-k} q^{k(n-k)}$ in the $k$-th row and $(n-k)$-th column and zeros otherwise. (We enumerate rows and columns from 0 to $n$.) Let $B(n)$ be the upper triangular matrix

$$
B(n)=\left(\begin{array}{ccccc}
\gamma_{0} & {\left[\begin{array}{c}
n \\
1
\end{array}\right] \gamma_{1}} & {\left[\begin{array}{c}
n \\
2
\end{array}\right] \gamma_{2}} & \cdots & \gamma_{n} \\
& \gamma_{0} & {\left[\begin{array}{c}
n-1 \\
1
\end{array}\right] \gamma_{1}} & \cdots & \gamma_{n-1} \\
& & \cdots & \cdots & \cdots \\
& & & \gamma_{0} & \gamma_{1} \\
& & & & \gamma_{0}
\end{array}\right)
$$

Thus the $(n-k)$-th row of $B(n)$ is

$$
0, \ldots, 0,\left[\begin{array}{l}
k \\
0
\end{array}\right] \gamma_{0},\left[\begin{array}{l}
k \\
1
\end{array}\right] \gamma_{1},\left[\begin{array}{l}
k \\
2
\end{array}\right] \gamma_{2}, \ldots,\left[\begin{array}{c}
k \\
k-1
\end{array}\right] \gamma_{k-1},\left[\begin{array}{l}
k \\
k
\end{array}\right] \gamma_{k}
$$

(6.7) Theorem. The matrix $A(n)$ is equal to the product $D(n) B(n)$.

Proof. The proof is by induction on $n$. We first compute the matrix of $\tau_{n, 1}$ on $V_{n} \otimes V_{1}$ and then restrict to $V_{n+1}$. In order to display the matrix of $\tau_{n, 1}$ we use the basis

$$
x_{0} \otimes x_{0}, \ldots, x_{n} \otimes x_{0}, x_{0} \otimes x_{1}, \ldots, x_{n} \otimes x_{1}
$$


The matrix of $\tau_{n, 1}$ has the block form

$$
\left(\begin{array}{cc}
0 & \beta A(n) \\
\alpha A(n) & A^{\prime}(n)
\end{array}\right)
$$

The matrix $A^{\prime}(n)$ is obtained from $A(n)$ in the following manner: Let $\alpha_{0}, \ldots, \alpha_{n}$ denote the columns of $A(n)$ and $\beta_{0}, \ldots, \beta_{n}$ the columns of $A^{\prime}(n)$. We claim that

$$
\beta_{i}=\alpha q^{2 i-n} \theta \alpha_{i}+\beta q^{2 i-n-1} \delta[n-i+1] \alpha_{i-1}+\alpha \delta[i+1] \alpha_{i+1}
$$

with $\alpha_{-1}=\alpha_{n+1}=0$.

Recall that $\tau_{n, 1}=\left(\tau_{n} \otimes 1\right) z_{1, n}\left(\tau_{1} \otimes 1\right) z_{n, 1}$. In our case the universal $R$-matrix has the simple form

$$
R=\kappa \circ\left(1+\left(q-q^{-1}\right) F \otimes E\right)
$$

For the convenience of the reader we display the four steps in the calculation of $\tau_{n, 1}$, separately for $x_{i} \otimes x_{0}$ and $x_{i} \otimes x_{1}$.

$$
\begin{aligned}
x_{i} \otimes x_{0} \mapsto & q^{(n-2 i) / 2} x_{0} \otimes x_{i} \\
\mapsto & \alpha q^{(n-2 i) / 2} x_{1} \otimes x_{i} \\
\mapsto & \alpha x_{i} \otimes x_{1} \\
\mapsto & \sum_{j} \alpha \alpha_{i}^{j} x_{j} \otimes x_{0} . \\
x_{i} \otimes x_{1} \mapsto & q^{-(n-2 i) / 2} x_{1} \otimes x_{i}+\delta[i+1] q^{(n-2 i-2) / 2} x_{0} \otimes x_{i+1} \\
\mapsto & q^{-(n-2 i) / 2}\left(\beta x_{0}+\theta x_{1}\right) \otimes x_{i}+\alpha \delta[i+1] q^{(n-2 i-2) / 2} x_{1} \otimes x_{i+1} \\
\mapsto & \beta x_{i} \otimes x_{0}+\beta q^{-n+2 i-1} \delta[n-i+1] x_{i-1} \otimes x_{1} \\
& +q^{2 i-n} \theta x_{i} \otimes x_{1}+\alpha \delta[i+1] x_{i+1} \otimes x_{1} \\
\mapsto & \sum_{j} \alpha_{i}^{j} x_{i} \otimes x_{0}+\sum_{j} \beta q^{2 i-n+1} \delta[n-i+1] \alpha_{i-1}^{j} x_{j} \otimes x_{1} \\
& +\sum_{j} q^{2 i-n} \theta \alpha_{i}^{j} x_{j} \otimes x_{1}+\sum_{j} \alpha \delta[i+1] \alpha_{i+1}^{j} x_{j} \otimes x_{1} .
\end{aligned}
$$

This proves the claim about the matrix for $\tau_{n, 1}$.

We now use the following fact about the Clebsch-Gordan decomposition (it is easily verified in our case, but see e.g. [7, VII.7] for more general results): In the Clebsch-Gordan decomposition $V_{n} \otimes V_{1}=V_{n+1} \oplus V_{n-1}$ a basis of $V_{n+1}$ is given by

$$
y_{j}=\frac{F^{j}}{[j] !}\left(x_{0} \otimes x_{0}\right)=q^{-j} x_{j} \otimes x_{0}+x_{j-1} \otimes x_{1}
$$


We apply $\tau_{n, 1}$ to the $y_{j}$. Since there are no overlaps between the coordinates of the $y_{j}$, we can directly write $\tau_{n, 1}\left(y_{j}\right)$ as a linear combination of the $y_{k}$.

We assume inductively that $A(n)$ has bottom-right triangular form (i.e., zeros above the codiagonal) with codiagonal as specified by $D(n)$. Then $A^{\prime}(n)$ has a nonzero line one step above the codiagonal and is bottom-right triangular otherwise. From the results so far we see that the columns of $A(n+1)$, enumerated from 0 to $n+1$, are obtained inductively as follows: The 0 -th row is $\left(0, \ldots, 0, \beta^{n+1}\right)$. Below this 0 -th row the $j$-th column, for $0 \leq j \leq n+1$, has the form

$$
\alpha q^{j} \alpha_{j}+q^{2 j-n-2} \theta \alpha_{j-1}+\beta q^{2 j-n-3} \delta[n-j+2] \alpha_{j-2} .
$$

From this recursive formula one derives immediately that the codiagonal of $A(n)$ is given by $D(n)$.

Finally, we prove by induction that $A(n)$ is as claimed. The element in row $k$ and column $n-k+j$ equals

$$
\alpha^{k} \beta^{n-k} q^{k(n-k)}\left[\begin{array}{l}
k \\
j
\end{array}\right] \gamma_{j}
$$

For $n=1$, we have defined $\tau_{1}$ as $A(1)$. For the inductive step we use (6.8) in order to determine the element of $A(n)$ in column $n-k+j$ and row $k+1$. The assertion is then equivalent to the following identity:

$$
\begin{aligned}
& \alpha^{k} \beta^{n-k} q^{k(n-k)}\left(\alpha\left[\begin{array}{l}
k \\
j
\end{array}\right] \gamma_{j}+q^{n-2 k+2 j-2} \theta\left[\begin{array}{c}
k \\
j-1
\end{array}\right] \gamma_{j-1}\right. \\
& \left.+\beta q^{n-2 k+2 j-3} \delta[k-j+2]\left[\begin{array}{c}
k \\
j-2
\end{array}\right] \gamma_{j-2}\right) \\
& =\alpha^{k+1} \beta^{n-k} q^{(n-k)(k+1)}\left[\begin{array}{c}
k+1 \\
j
\end{array}\right] \gamma_{j} \text {. }
\end{aligned}
$$

We cancel $\alpha$-, $\beta$-, and $q$-factors, use the Pascal formula

$$
\left[\begin{array}{c}
a+1 \\
b
\end{array}\right]=q^{b}\left[\begin{array}{l}
a \\
b
\end{array}\right]+q^{-a+b-1}\left[\begin{array}{c}
a \\
b-1
\end{array}\right]
$$

and the identity

$$
[k-j+2]\left[\begin{array}{c}
k \\
j-2
\end{array}\right]=\left[\begin{array}{c}
k \\
j-1
\end{array}\right][j-1]
$$

and see that the identity in question is equivalent to the recursion formula (6.5) defining the $\gamma$-polynomials. 
We formulate the main result of this section in a different way. First we note that it was not essential to work with the function field $\mathcal{R}$. In fact $\boldsymbol{R}$ could have been any commutative ring and $q, \alpha, \beta$ could have been any suitable parameters in it. We think of $\theta$ as being an indeterminate.

Let $L(\alpha, \beta)$ be the operator on integrable $U$-modules which acts on $V_{n}$ via

$$
x_{j} \mapsto \alpha^{n-j} \beta^{j} q^{j(n-j)} x_{n-j} .
$$

Let

$$
T(\alpha, \beta)=\sum_{k=0}^{\infty} \gamma_{k} \frac{E^{k}}{[k] !}
$$

$T(\alpha, \beta)$ is well-defined as an operator on integrable $U$-modules. Then (6.7) can be expressed as follows:

(6.11) Theorem. The operator $t(\alpha, \beta)=L(\alpha, \beta) \circ T(\alpha, \beta)$ acts on $V_{n}$ as $\tau_{n}$.

In section 8 we give another derivation of this operator from the universal point of view.

One can develop a parallel theory by starting with the four braid pair $\left(X^{-1}, F^{-1}\right)$. This leads to matrices which are top-left triangular (i. e., zeros below the codiagonal). By computing the inverse of (5.1) and (5.2) we see that, in the case $(\alpha, \beta)=(1,1)$, we have to replace $(q, \theta)$ by $\left(q^{-1},-\theta\right)$.

The following proposition may occasionally be useful. Introduce a new basis $u_{0}, \ldots, u_{n}$ in $V_{n}$ by

$$
x_{i}=q^{-i(n-i) / 2} \sqrt{\left[\begin{array}{c}
n \\
i
\end{array}\right]} u_{i}
$$

Then a little computation shows:

(6.12) Proposition. Suppose $\alpha=\beta$. With respect to the basis $\left(u_{i}\right)$ the $R$-matrix and the matrix for $\tau_{n}$ are symmetric.

\section{The $\gamma$-polynomials}

For later use we derive some identities for the $\gamma$-polynomials of the previous section. A basic one comes from the compatibility of the cylinder twist with tensor products. Again we use $\delta=q-q^{-1}$. We give two proofs of (7.1).

(7.1) Theorem. The $\gamma$-polynomials satisfy the product formula

$$
\gamma_{m+n}=\sum_{k=0}^{\min (m, n)} \alpha^{-k} \beta^{k} q^{m n-k(k+1) / 2} \delta^{k}[k] !\left[\begin{array}{l}
m \\
k
\end{array}\right]\left[\begin{array}{l}
n \\
k
\end{array}\right] \gamma_{m-k} \gamma_{n-k} .
$$


Proof. For the proof we consider $\tau_{m, n}=\left(\tau_{m} \otimes 1\right) z_{n, m}\left(\tau_{n} \otimes 1\right) z_{m, n}$ on $V_{m} \otimes V_{n}$ and compute the coefficient of $x_{m} \otimes x_{n}$ in $\tau_{m, n}\left(x_{m} \otimes x_{n}\right)$. Note that $x_{m} \otimes x_{n}$ is the $F$ primitive vector (lowest weight vector) of the summand $V_{m+n} \subset V_{m} \otimes V_{n}$ in the standard basis. Hence the coefficient in question is $\alpha_{m+n}^{m+n}(m+n)$ which, by (6.7), equals $\alpha^{m+n} \gamma_{m+n}$.

From the form of the universal $R$-matrix we see directly that $z_{m, n}\left(x_{m} \otimes x_{n}\right)=$ $q^{m n / 2} x_{n} \otimes x_{m}$. If we write

$$
z_{n, m}\left(x_{j} \otimes x_{m}\right)=\sum_{u, v} r_{j m}^{u v} x_{u} \otimes x_{v},
$$

then

$$
\tau_{m, n}\left(x_{m} \otimes x_{n}\right)=\sum_{j, k, u, v} q^{m n / 2} \alpha_{n}^{j}(n) r_{j m}^{u v} \alpha_{u}^{k}(m) x_{k} \otimes x_{v}
$$

We need the coefficient when $(k, v)=(m, n)$ in which case we have the formal identity

$$
\alpha^{m+n} \gamma_{m+n}=\sum_{j, u} q^{m n / 2} \alpha_{n}^{j}(n) r_{j m}^{u n} \alpha_{u}^{m}(m)
$$

The universal $R$-matrix yields

$$
z_{n, m}\left(x_{j} \otimes x_{m}\right)=\sum_{k \geq 0} v^{\bullet} \delta^{k}[j+1] \cdots[j+k] x_{m-k} \otimes x_{j+k}
$$

with $\cdot=k(k-1) / 2+(n-2 j-2 k)(2 k-m) / 2$. Moreover

$$
\alpha_{n}^{n-k}(n)=\alpha^{n-k} \beta^{k} q^{(n-k) k} \gamma_{n-k}, \quad \alpha_{m-k}^{m}(m)=\alpha^{m}\left[\begin{array}{c}
m \\
k
\end{array}\right] \gamma_{m-k} .
$$

When we insert these values into the formal equation for $\alpha^{m+n} \gamma_{m+n}$, we obtain the desired result.

The dependence of $\gamma_{k}$ on the parameters $\alpha$ and $\beta$ is not essential. Define inductively polynomials $\gamma_{k}^{\prime}$ in $\theta$ over $\mathbb{Z}\left[q, q^{-1}\right]$ by setting $\gamma_{-1}^{\prime}=0, \gamma_{0}^{\prime}=1$ and, for $k \geq 0$,

$$
\gamma_{k+1}^{\prime}=q^{k} \theta \gamma_{k}^{\prime}+q^{k-1} \delta[k] \gamma_{k-1}^{\prime},
$$

i.e., by setting $\gamma_{k}^{\prime}(\theta, q)=\gamma_{k}(\theta, q, 1,1)$. A simple rewriting of the recursion formulas then yields the identity

$$
\gamma_{k}(\theta, q, \alpha, \beta)=\gamma_{k}^{\prime}\left(\frac{\theta}{\sqrt{\alpha \beta}}, q\right)\left(\frac{\beta}{\alpha}\right)^{k / 2} .
$$

Note that $\gamma_{k}^{\prime}$ contains only powers $\theta^{l}$ with $l \equiv k \bmod 2$. 
Normalize the $\gamma^{\prime}$ to obtain monic polynomials $\beta_{k}(\theta)=q^{-k(k-1) / 2} \gamma_{k}^{\prime}(\theta)$. The new polynomials satisfy the recursion relation

$$
\beta_{k+1}=\theta \beta_{k}+\left(1-q^{-2 k}\right) \beta_{k-1}
$$

In order to find an explicit expression for the $\beta_{k}$, we introduce a new variable $\varrho$ via the quadratic relation $\theta=\varrho-\varrho^{-1}$.

(7.4) Proposition. The polynomials $\beta$ satisfy the identity

$$
\beta_{n}\left(\varrho-\varrho^{-1}\right)=\sum_{j=0}^{n}(-1)^{j} q^{-j(n-j)}\left[\begin{array}{l}
n \\
j
\end{array}\right] \varrho^{n-2 j}
$$

Proof. We use this identity in the recursion formula (7.3) and compare the coefficients of $\varrho^{n+1-2 j}$. A little rewriting shows that the claim reduces to the Pascal formula (6.9) for the $q$-binomial coefficients.

We can write $\varrho^{k}+(-1)^{k} \varrho^{-k}$ as an integral polynomial $P_{k}$ in $\theta=\varrho-\varrho^{-1}$. That polynomial satisfies the recursion relation

$$
\theta P_{k}=P_{k+1}-P_{k-1}
$$

It is possible to write $P_{k}$ in terms of Tschebischev- or Jacobi-polynomials. The last proposition thus gives

$$
\beta_{n}(\theta)=\sum_{j=0}^{[n / 2]}(-1)^{j} q^{-j(n-j)}\left[\begin{array}{l}
n \\
j
\end{array}\right] P_{n-2 j}(\theta)
$$

The product formula (7.1) was a consequence of representation theory. In view of the applications to be made in section 8 it is desirable to have a proof which uses only the recursive definition of the $\gamma$-polynomials. We now give such a proof. By (7.2), it suffices to consider the case $\alpha=\beta=1$.

Second proof of (7.1). We write

$$
C_{k}^{m, n}=q^{m n-k(k+1) / 2} \delta^{k}[k] !\left[\begin{array}{l}
m \\
k
\end{array}\right]\left[\begin{array}{l}
n \\
k
\end{array}\right],
$$

and want to show that

$$
\gamma_{m+n}=\sum_{k=0}^{\min (m, n)} C_{k}^{m, n} \gamma_{m-k} \gamma_{n-k}
$$


Denote the right hand side by $\gamma(m, n)$. Then $\gamma(m, n)=\gamma(n, m)$. We will use the recursion (6.5) and the Pascal formula (6.9), with $q$ replaced by $q^{-1}$, to show $\gamma(m+1, n)=\gamma(m, n+1)$. Since $\gamma(m+n, 0)=\gamma_{m+n}$ the proof will then be complete. We set $\gamma_{k}=0$ for $k<0$, then we can sum just over $k \geq 0$. The $C$-coefficients satisfy the following Pascal type relation

$$
C_{k}^{m+1, n}=q^{n-k} C_{k}^{m, n}+\delta q^{n-k+1} q^{m-k}[n-k+1] C_{k-1}^{m, n} .
$$

The verification of (7.5) uses the Pascal formula for $\left[\begin{array}{c}m+1 \\ k\end{array}\right]$ and a little rewriting. We now apply this relation in the sum $\gamma(m+1, n)$ and obtain (with an index shift $k \rightarrow k+1$ in the second summand)

$$
\begin{aligned}
\gamma(m+1, n)= & \sum_{k} q^{n-k} C_{k}^{m, n} \gamma_{m-k+1} \gamma_{n-k} \\
& +\sum_{k}\left(\delta[n-k] q^{n-k-1} \gamma_{n-k-1}\right) q^{m-k} C_{k}^{m, n} \gamma_{m-k}
\end{aligned}
$$

In the second sum we apply the recursion to the factor in parentheses to obtain

$$
\begin{aligned}
& \gamma(m+1, n) \\
& =\sum_{k} C_{k}^{m, n}\left(q^{n-k} \gamma_{m-k+1} \gamma_{n-k}+q^{m-k} \gamma_{m-k} \gamma_{n-k+1}-q^{n+m-2 k} \theta \gamma_{m-k} \gamma_{n-k}\right) .
\end{aligned}
$$

Since $\gamma(m, n)=\gamma(n, m)$, we obtain $\gamma(m, n+1)$ from $\gamma(m+1, n)$ upon interchanging $m$ and $n$ in the foregoing identity. That interchanges the first two summands in the parentheses and leaves the third fixed.

\section{The universal cylinder twist}

In this section we work with operators on integrable $U$-modules. These are $\Omega$-linear weak endomorphisms of the category $U$-Int. Left multiplication by $x \in U$ is such an operator; it will be denoted by the same symbol or by $l_{x}$. If $t$ is an operator, then $\mu(t)$ is the operator on $U$-Int $\times U$-Int which is given by the action of $t$ on tensor products of modules. If $\tau$ denotes the twist operator, then we define $\tau(t)=\tau \circ t \circ \tau$. We have the compatibility $\mu\left(l_{x}\right)=l_{\mu(x)}$ and $\tau \mu\left(l_{x}\right)=l_{\tau \mu(x)}$. The operators $\mu(t)$ and $\tau(t)$ are again weak endomorphisms of the categories involved.

Typical examples of such operators which are not themselves elements of $U$ are the universal $R$-matrix $R$ and its factors $\kappa$ and $\Psi$, see (6.1). We also use the operators $L=T_{i, 1}^{\prime}$ and $L^{\#}=T_{i, 1}^{\prime \prime}$ of Lusztig [8, p. 42].

Since $R$ acts by $U$-linear maps each operator $t$ satisfies the standard relation

$$
R \circ \mu(t)=\tau \mu(t) \circ R
$$

of a braiding. 
An operator $t$ is called a universal cylinder twist on $U$-Int if it is invertible and satisfies the analogue of (1.4)

$$
\begin{aligned}
& \mu(t)=\tau R(1 \otimes t) R(t \otimes 1) \\
& \tau R(1 \otimes t) R(t \otimes 1)=(t \otimes 1) \tau R(1 \otimes t) R
\end{aligned}
$$

We denote by $t_{V}$ the action of $t$ on the module $V$. Then (1.3) holds if we use $R$ to define the braiding. Recall the operator $t(\alpha, \beta)$, defined at the end of section 6 . Here is the main result:

(8.4) Theorem. Suppose $\alpha \beta=-q$. Then $t(\alpha, \beta)$ is a universal cylinder twist.

We treat the case $(\alpha, \beta)=(1,-q)$ in detail and reduce the general case formally to this one. We skip the notation $\alpha, \beta$ and work with $t=L T$. Note that $L$ is Lusztig's operator referred to above. We collect a few properties of $L$ in the next lemma.

(8.5) Lemma. The operator $L$ satisfies the following identities:

(1) $L E L^{-1}=-K F, L F L^{-1}=-E K^{-1}, L K L^{-1}=K^{-1}$.

(2) $\mu(L)=(L \otimes L) \Psi=\tau R(L \otimes L) \kappa^{-1}$.

(3) $\kappa(L \otimes 1)=(L \otimes 1) \kappa^{-1}, \kappa(1 \otimes L)=(1 \otimes L) \kappa^{-1}$.

(4) $(L \otimes L) \Psi(L \otimes L)^{-1}=\kappa \circ \tau \Psi \circ \kappa^{-1}$.

Proof. For (1) see [8, Proposition 5.2.4.]. A simple computation from the definitions yields (3) and (4). For the first equality in (2), in the case $L^{\#}$ see [8, Proposition 5.3.4]; the second one follows by using (3) and (4).

In the universal case one of the axioms for a cylinder twist is redundant:

(8.6) Proposition. If the operator $t$ satisfies (8.2) then it also satisfies (8.3).

Proof. Apply $\tau$ to (8.2) and use (8.1).

Proof of theorem (8.4). The operator $L$ is invertible. The operator $T$ is invertible since its constant term is 1 . Thus it remains to verify (8.2). We show that this identity is equivalent to

$$
\mu(T)=\kappa(1 \otimes T) \kappa^{-1} \circ\left(L^{-1} \otimes 1\right) \Psi(L \otimes 1) \circ(T \otimes 1)
$$

given the relations of Lemma (8.5). Given (8.2), we have

$$
\mu(T)=\mu\left(L^{-1}\right) \tau(R)(1 \otimes L T) \kappa \Psi(L T \otimes 1)
$$


We use (8.5.2) for $\mu\left(L^{-1}\right)$, cancel $\tau(R)$ and its inverse, and then use (8.5.3); (8.7) drops out. Similarly backwards.

In order to prove (8.7), one verifies the following identities from the definitions

$$
\begin{aligned}
& \kappa(1 \otimes T) \kappa^{-1}=\sum_{k=0}^{\infty} \frac{\gamma_{k}}{[k] !}\left(K^{k} \otimes E^{k}\right) \\
& \left(L^{-1} \otimes 1\right) \Psi(L \otimes 1)=\sum_{k=0}^{\infty}\left(-1^{k} q^{-k(k-1) / 2} \frac{\delta^{k}}{[k] !} K^{k} E^{k} \otimes E^{k}\right.
\end{aligned}
$$

Using this information, we compute the coefficient of $K^{r} E^{s} \otimes E^{r}$ on the right hand side of $(8.7)$ to be

$$
\sum_{n=0}^{\min (r, s)}(-1)^{n} q^{-n(n-1) / 2} \frac{\delta^{n}}{[n] ![s-n] ![r-n] !} \gamma_{s-n} \gamma_{r-n}
$$

The coefficient of the same element in $\mu(T)$ is, by the $q$-binomial formula, equal to

$$
q^{-r s} \frac{1}{[s] ![r] !} \gamma_{r+s}
$$

Equality of these coefficients is exactly the product formula (7.1) in the case where $(\alpha, \beta)=(1,-q)$. This finishes the proof of the theorem in this special case.

A similar proof works in the general case. A formal reduction to the special case uses the following observation. Write $\alpha=q^{-\zeta}$. Then, formally, $L(\alpha, \beta)=K^{\zeta} L$ in case $\alpha \beta=-q$. This fact is used to deduce similar properties for $L_{\#}=L(\alpha, \beta)$ from lemma (8.5), in particular

$$
L_{\#}^{-1} F L_{\#}=\alpha^{-1} \beta q K E .
$$

The final identity leads to (7.1) in the general case.

We point out that the main identity in the construction of the universal twist involves only the Borel subalgebra of $U$ generated by $E, K$. Of course, there is a similar theory based on $F, K$ and another braiding. The constructions of section 6 show that the universal twist is determined by its action on the 2-dimensional module $V_{1}$. Hence our main theorem gives all possible universal cylinder twists associated to the given braided category $U$-Int.

\section{References}

[1] Bourbaki, N.: Groupes et algèbres de Lie. Chapitres 4, 5 et 6. Paris, Hermann 1968

[2] Brieskorn, E.: Die Fundamentalgruppe des Raumes der regulären Orbits einer endlichen komplexen Spiegelungsgruppe. Inv. Math. 12 (1971), 57-61 
[3] tom Dieck, T.: Symmetrische Brücken und Knotentheorie zu den Dynkin-Diagrammen vom Typ B. J. Reine Angew. Math. 451 (1994), 71-88

[4] tom Dieck, T:: On tensor representations of knot algebras. Manuscripta Math. 93 (1997), $163-176$

[5] tom Dieck, T., Häring-Oldenburg, R.: Tensor categories with cylinder braiding. In preparation

[6] Häring-Oldenburg, R.: Dissertation Göttingen 1998

[7] Kassel, C.: Quantum groups. Berlin, Springer 1995

[8] Lusztig, G.: Introduction to quantum groups. Basel, Birkhäuser 1993

[9] Turaev, V.G.: Quantum invariants of knots and 3-manifolds. Berlin, W. de Gruyter 1994

Received December 9, 1996

Tammo tom Dieck, Mathematisches Institut, Bunsenstraße 3/5, D-37073 Göttingen tammo@cfgauss.uni-math.gwdg.de

Reinhard Häring-Oldenburg, Mathematisches Institut, Bunsenstraße 3/5, D-37073 Göttingen haering@cfgauss.uni-math.gwdg.de 
